\title{
Promoting Oral Hygiene Behavior in Recruits in the Dutch Army
}

\author{
Yvonne A. B. Buunk-Werkhoven, MSc, $R D H^{*}$ S; Arie Dijkstra, PhDt; \\ Lt-Col Henk van der Wal, MBA-H, MHA, EMSD‡; Nina Basic, RDH§; PFC Steven A. Loomans, RDH§; \\ Cees P. van der Schans, PT, CE, PhD*; Brig-Gen Rob van der Meer, Surgeon Generalł
}

\begin{abstract}
Objectives: To make practical recommendations for improving oral hygiene behavior (OHB) potential predictors based on the Theory of Planned Behavior (TPB) were assessed. Measurements of oral health knowledge $(\mathrm{OHK})$ and the expected social effect for having healthy teeth were included. Methods: 216 recruits in the Dutch Army ground forces completed a questionnaire about oral hygiene behavior, attitudes, social norms, perceived behavioral control (PBC), intention to perform optimal $\mathrm{OHB}, \mathrm{OHK}$, and expected social outcomes. Results: The multivariate regression analysis revealed that attitude and $\mathrm{PBC}$ explained $37.2 \%$ of the variance in intention to perform optimal oral hygiene behavior, which is a substantial proportion. Furthermore, actual oral hygiene behavior was only predicted by attitude, explaining $7.1 \%$ of the variance. Conclusion: The present findings suggest that recruits' oral hygiene behavior may be improved by promoting a more positive attitude and especially by enhancing perceived behavior control.
\end{abstract}

\section{INTRODUCTION}

To maintain good oral health, adherence to optimal oral hygiene behavior is required. Despite the importance of oral hygiene, which is widely acknowledged, it seems to be a problem for military recruits to perform oral hygiene practices in an appropriate and efficient manner. For example, in a study of 912 members of the Croatian Army it was shown that oral health of the examined soldiers was in general poor, as a consequence of insufficient oral hygiene. ${ }^{1}$

The Netherlands Armed Forces has a complete health care system at its disposal. The first line military health care is provided by clinics at the home units and operational medical units during training and deployments. In both situations integrated health care is provided, with a combination of general practice medicine, preventive medicine, and occupational health services. ${ }^{2}$ Oral health care is part of this integrated health care system. As for recruits who are selected to be deployed, dental fitness is one of the most important conditions. Before deployment, a soldier has to comply with a NATO agreed mandatory minimum level of sufficient oral health care, called Dental Fit

*Research Group in Health Care and Nursing, Hanze University Applied Sciences, Groningen Eyssoniusplein 18, Postbus 3109, 9701 DC, Groningen, The Netherlands.

$\dagger$ Department of Social and Organizational Psychology, University of Groningen, Grote Kruisstraat 2/1, 9721 TS, Groningen, The Netherlands.

$\ddagger$ Ministry of Defence, Office of the Surgeon General, Binckhorstlaan 135, 2516 BA, The Hague, The Netherlands.

$\S$ Center for Dentistry and Oral Hygiene, Hanze University Applied Sciences, Groningen, Department of Oral Health Care, University Medical Center Groningen, University of Groningen, Antonius Deusinglaan 1, 9713 AV, Groningen, The Netherlands.

Previous poster presentation: "Promoting Oral Health Self-Care in Recruits in the Dutch Army" at the first annual 2008 AMSUS International Delegates Poster Session in San Antonio, Texas (November 9-14, 2008).

This manuscript was received for review in December 2008. The revised manuscript was accepted for publication in June 2009.

Reprint \& Copyright (C) by Association of Military Surgeons of U.S., 2009. class 1 or 2 (according to Standard NATO Agreement 2466 "Dental fitness standards for military personnel and a dental fitness classification system"). Thus far it is not clear what the actual prevalence of oral diseases among Dutch recruits is, but results from an earlier study in The Netherlands showed that only one out of approximately 2,000 military recruits (18-20 years old) appeared to be absolutely free of caries experience. ${ }^{3}$

Professionals in oral health care have recognized that assessments of oral health and oral hygiene outcomes are of great importance for developing oral health care interventions. In addition, oral self-care based on recruits' personal choice may be considered as an important aspect of oral hygiene behavior. Therefore, individual beliefs and attitudes toward optimal oral hygiene behavior may play an important role in the maintenance of good oral health. In turn, good oral health may lead to a reduced number of dental interventions and omissions during training and deployments.

In the present study, the Theory of Planned Behavior (TPB), which is the model most often used to map the psychological causes of health behaviors, was used to predict psychological determinants of oral hygiene behavior. The TPB includes 3 psychological factors as independent determinants of behavioral intention, which in turn influences subsequent behavior: ${ }^{4,5}$

(1) Attitude (i.e., a person's positive or negative feelings about a given behavior, for example "I hate brushing my teeth twice a day, and cleaning interdentally at least once a day").

(2) Subjective norm (i.e., the belief that specific important persons think that one should or should not perform a given behavior, for example, "My dentist thinks that I should brush my teeth twice a day, and use interdental aids at least once a day").

(3) Perceived behavior control (i.e., a person's perception of his/her capabilities to perform a behavior, for example 
"I think I will be able to brush my teeth twice a day, and use interdental aids at least once a day").

Overall, for oral hygiene behavior, the TPB suggests that the more positive the attitude toward oral hygiene practices, the stronger the social norms, and the higher the perceived behavior control, the more likely it is that an individual will perform an optimal oral hygiene behavior. ${ }^{4,5}$ The predictive utility of the TPB has been supported in investigations of a wide range of behaviors. It has been reported that intention is the strongest psychological predictor of behavior, and metaanalyses show that across studies the average explained variance in behavior is $22 \% .^{6,7}$

In addition, according to the TPB model, individuals make rational decisions based in part on their oral health knowledge; this variable was assessed too. However, health-related concerns are probably not the only motive for oral hygiene behavior. Indeed, in a study among girls, it was suggested that behaviors that may promote health are often performed for reasons other than improvements in general health; for example, tooth brushing may be engaged in to look more attractive. ${ }^{8}$ In a similar vein, it has also been suggested that unhealthy teeth are perceived as negatively affecting a person's image. ${ }^{9}$ Therefore, the expected social outcomes of having healthy teeth were assessed too.

The relevance of the behavioral sciences for modifying individual oral hygiene behavior has been shown since the early seventies and from that moment the behavioral and social sciences were definitively linked with dentistry in the Fédération Dentaire International's publication of Social Sciences and Dentistry. ${ }^{10}$ For instance, the findings of a study among 214 participants, adequate oral hygiene behavior (tooth brushing and the use of interdental cleaning aids) was associated with an individual's attitude toward oral health - "clean teeth" and "fresh breath"and with the perceived influence of "important others."

\section{Overview Present Research}

The aim of the present study was to identify the relevant predictors of optimal oral hygiene behavior. These potential predictors were the variables specified in the TPB. In addition, measures of oral health knowledge and of expected outcomes of having healthy teeth for one's interpersonal relationships were used as predictors.

\section{METHODS}

After obtaining official authorization from the commanding officers in charge for participation of the army unit in this study, the adminstration of a customized questionnaire was linked to the mandatory vaccination program in July, October, and November 2007. The recruits of the Schoolbat North of the post "J. W. F. Kazerne" were asked if they were willing to participate in the study on a voluntary basis. They were free to refuse participation, and no pressure was exerted to take part in the study. Therefore written informed consent was waived and only verbal informed consent obtained.

\section{Measures}

The questionnaire included 58 items divided into several parts, including a few demographic questions on matters such as age, nationality, marital status, and education. Level of education was categorized as low, medium, or high. In The Netherlands, low educational level refers to vocational training, medium level to advanced vocational training, and high level to college/university training. These and other items about dental history, experiences, and dental health status were open ended, multiple choice, or to be answered on bipolar adjective rating scales.

Oral hygiene behavior (OHB) was measured by using an index for oral hygiene behavior (OHB index) developed by Buunk-Werkhoven, Dijkstra, and van der Schans, 2009 (submitted for publication). The index includes 8 items with respect to tooth brushing, interdental cleaning, and tongue cleaning (Appendix 1). For example, the item "I brush my teeth as follows" was supported by pictures showing different brushing methods such as horizontal, vertical, circular, and the Bass method. After the item scores were assigned weights, the index values were calculated and a sum score was computed. The OHB sum score on this index could range from 0 to 16. A high sum score indicate a high level of adequate oral hygiene behavior.

After the recruits had filled in the OHB index, in which they had reported their actual oral hygiene behavior, an elaborate description of a daily regimen of optimal oral hygiene behavior recommended by the American Dental Association (ADA) was given on paper. ${ }^{12}$

Optimal oral hygiene behavior was described as "brushing your teeth twice a day (once after breakfast and once before going to sleep), using a soft-bristled toothbrush and fluoride-containing toothpaste; brushing softly/without pressure for at least 2 minutes; brushing stepwise by making small strokes - sort of massage - near the gum, along the inside and the outside, and on the jackdaw areas. In addition to the tooth brushing, daily interdental cleaning, (i.e., the use of floss, tooth sticks, or interdental brushes at least once a day), and tongue cleaning is also recommended."

Directly after this description recruits were asked if they performed this recommended oral hygiene behavior, and the answer could be scored with 1 , yes or 0 , no.

Intention to perform optimal oral hygiene behavior was measured using a sum score constructed from 2 items, e.g., "Do you intend to perform optimal oral hygiene behavior as described, within the next 6 months?," which was answered with endpoints 1 , absolutely not to 7, absolutely yes, and "Is it likely that you will start to perform optimal oral hygiene behavior as described, within the next 6 months?" with the endpoints 1 , totally unlikely to 7 , totally likely. The intention sum score could range from 2 to 14 .

\section{Variables of TPB}

In accordance with the TPB, the recruits' attitudes, social norms, and perceived behavioral control of both their intention 
to perform adequate oral self-care and their actual oral hygiene were assessed using a total of 17 items.

Attitude toward optimal oral hygiene behavior were measured using 9 worded statements in a semantic differential format. The recruits indicated on 7-point scales how they evaluated this advised oral hygiene behavior, on the dimensions 1, unimportant to 7, important, etc.,: unpleasant/pleasant, unhealthy/healthy, negative/positive, annoying/not annoying, not useful/useful, boring/exciting, painful/painless, and stu$\mathrm{pid} / \mathrm{smart}$. A sum score for attitudes, ranging from 9 to 63 , was constructed by adding these items. Higher scores indicate a more positive attitude.

Social norms toward the focal oral hygiene behavior were assessed by having the recruits rate the perceived opinions of 5 different significant others with respect to taking better care of their teeth, e.g., "my dentist," "my partner," "my family (parents, brothers, and sisters)," "my friends," and "my colleagues." The 7-point scale for social norms ( $\mathrm{SN}$ ) was based on 5 items, and a sum score on this SN scale varied from 5 to 35 .

Perceived behavioral control (PBC) was measured using a sum score constructed from 3 items, e.g., "If I wanted to, I could take care of my teeth as described," which was answered with endpoints 1, don't agree to 5, agree or "I find it difficult or easy to take care of my teeth based the described optimal oral hygiene behavior," with the endpoints 1 , difficult to 5 , easy. The sum score on the PBC 5-point scale ranged from 3 to 15 . In all 3 cases, high sum scores indicated a positive attitude, strong perceived approval from significant others, and a high level of perceived behavioral control of the intention to perform optimal oral hygiene behavior.

Expected social outcomes (ESO) for having healthy teeth included 6 items. An example of this 5-point scale is: "In social contacts fresh breath is important." Responses varied from 1, disagree to 5, agree, and a sum score (ranging from 6 to 30 ) was computed by summing up scores on all 6 items that measured the concept ESO.

Oral health knowledge refers to the degree to which a recruit has sufficient or insufficient knowledge of oral health issues. The index consists of 16 items to reveal the status of the individual's oral health knowledge. All items could be scored with 1 , yes or 0 , no, and a sum score was computed, so that a total oral health knowledge score was formed for each recruit (ranging from 0 to 16). The higher the total score, the higher the individual's knowledge of oral health issues.

\section{Statistical Analysis}

The Statistical Package for Social Sciences 14.0 (SPSS, Chicago, Illinois) was used for data analysis. The internal consistency of the used scales was assessed by Cronbach's $\alpha$. Pearson correlations were calculated for univariate associations between the variables, and subsequently multivariate correlation analyses (linear regression analysis) were performed to identify the variables that accounted for a significant proportion of the variance in oral hygiene behavior.

\section{RESULTS}

A total of 216 (92\% male) recruits, with a mean (SD) age of 19 (2) years (ranging from 17 to 30 years) participated in the study. Ninety-five percent of the recruits were of Dutch nationality; they lived with their parents, and they had a medium $(48 \%)$ or a low (37\%) level of education.

The question regarding recruits' ability to perform optimal oral hygiene behavior as described was answered with 'yes' by 108 recruits $(50 \%)$. This subgroup performed this oral hygiene behavior for the average of 3 years, ranging from 2 months to 20 years. The other half of the total sample (49\%) did not perform optimal oral hygiene behavior as recommended. However, the reported results of the OHB index showed that around two-thirds of the recruits brushed their teeth as recommended by professionals; $2-3$ minutes (73\%) softly (69\%) twice a day (69\%), and $45 \%$ of the recruits brushed their teeth in the morning, and $83 \%$ before they go to sleep. In addition, $68 \%$ used fluoride-containing toothpaste, only $13 \%$ used interdental cleaning aids, and about $25 \%$ cleaned their tongue.

Table I shows that the Cronbach's $\alpha$ values of the used measures were moderate to good. Furthermore, the recruits' intention to perform optimal oral hygiene behavior is quite high, and, according to their attitude scores, they evaluated the recommended oral hygiene behavior positively. They attached a moderate value to the positive social outcomes of having healthy teeth, and their knowledge of oral health was good. Their scores on social norms indicated that the recruits reported some pressure from their social environment to perform optimal oral hygiene behavior. In addition, they felt they had considerable control over carrying out the oral self-care practices.

Correlational analyses were carried out to establish the direction and magnitude of the associations between the variables (see Table II). The recruits' actual oral hygiene behavior was found to correlate positively and significantly with attitude, perceived behavioral control, intention to perform oral hygiene behavior, and expected social outcomes. Moreover, oral hygiene behavior was negatively and not significantly associated with social norms, and also not significantly, but positively associated with oral health knowledge.

Finally, 2 linear regression analyses were performed to examine the multivariate relations of the TPB variables and

TABLE I. Cronbach's $\alpha$, Range, Means, and Standard Deviation (SD) for the Main Variables

\begin{tabular}{|lccc|}
\hline \multicolumn{1}{|c}{ Variablest } & Cronbach's $\alpha$ & $\begin{array}{c}\text { Mean (SD) } \\
\text { Range }\end{array}$ & (Whole Sample) \\
\hline Oral hygiene behavior $^{a}$ & - & $0-16$ & $10.08(2.32)$ \\
Intention to perform OHB $^{b}$ & 0.92 & $2-14$ & $9.62(3.59)$ \\
Attitude $^{c}$ & 0.91 & $9-63$ & $50.86(9.32)$ \\
Social norms $^{a}$ & 0.84 & $5-35$ & $11.99(6.38)$ \\
Perceived behavioral control $^{c}$ & 0.73 & $3-15$ & $11.82(2.79)$ \\
Expected social outcomes $^{d}$ & 0.79 & $6-30$ & $21.42(4.65)$ \\
Oral health knowledge $^{e}$ & - & $0-16$ & $13.18(1.50)$ \\
\hline
\end{tabular}

In the whole sample: ${ }^{a} n=210 ;{ }^{b} n=209 ;{ }^{c} n=208 ;{ }^{d} n=214 ;{ }^{e} n=206$. 
TABLE II. Correlations Between the Main Variables and Actual Oral Hygiene Behavior

\begin{tabular}{|lrrrrrrr|}
\hline \hline Variables & 1 & 2 & 3 & 4 & 5 & 6 & 7 \\
\hline $\begin{array}{l}\text { (1) Attitude } \\
\text { (2) Social norms }\end{array}$ & $-0.27^{* *}$ & - & & & & \\
(3) Perceived & $0.26^{* *}$ & -0.13 & - & & & \\
behavioral \\
control
\end{tabular}

$* p<0.05 ; * * p<0.001$

the 2 additional variables expected social outcomes and oral health knowledge, with intention to perform optimal oral hygiene behavior and subsequently with actual oral hygiene behavior (see Table III). Because this research focused primarily on the independent effects of the predictors, in line with the common procedure in research on the TPB, all variables were entered in a single step. In addition, the variances of predictor variables were sufficiently similar to include them in one model. The first regression analysis with intention as dependent variable proved to be significant $F(5,192)=24.36$, $p<0.001$ and accounted for $37.2 \%$ of the variance, which is a substantial proportion of the intention to perform the recommended oral hygiene behavior. Only perceived behavioral control and attitude emerged as significant predictors of the intention to perform this behavior. In these multivariate analyses, social norms, expected social outcomes, and knowledge were no longer significantly related to intention. Next, the second regression analysis with oral hygiene behavior as dependent variable proved to be significant too, $F(5,192)=$ $4.01, p<0.001$, but accounted for only $7.1 \%$ of the variance in actual oral hygiene behavior. Only attitude emerged as a

TABLE III. Linear Regression of Intention to Perform Optimal Oral Hygiene Behavior and Actual Oral Hygiene Behavior on TPB Variables, Expected Social Outcomes, and Oral Health Knowledge

\begin{tabular}{|c|c|c|}
\hline Determinants & $\begin{array}{c}\begin{array}{c}\text { Intention to Perform } \\
\text { Optimal Oral } \\
\text { Hygiene Behavior }\end{array} \\
\beta\end{array}$ & $\begin{array}{c}\begin{array}{c}\text { Actual Oral } \\
\text { Hygiene Behavior }\end{array} \\
\beta\end{array}$ \\
\hline Attitude & $0.25^{*}$ & $0.21 *$ \\
\hline Social norms & 0.05 & -0.01 \\
\hline $\begin{array}{l}\text { Perceived } \\
\quad \text { behavioral control }\end{array}$ & $0.51 *$ & 0.09 \\
\hline $\begin{array}{l}\text { Expected social } \\
\text { outcomes }\end{array}$ & 0.16 & 0.08 \\
\hline $\begin{array}{l}\text { Oral health } \\
\text { knowledge }\end{array}$ & 0.13 & 0.06 \\
\hline
\end{tabular}

$* p<0.001$. significant predictor of oral hygiene behavior. In this multivariate analysis, perceived behavioral control and expected social outcomes were no longer significantly related to oral hygiene behavior (see Table III for all $\beta \mathrm{s}$ ).

\section{DISCUSSION}

The findings of this study are particularly important as an elaborate OHB index was used that corresponds closely with what professionals consider optimal oral hygiene behavior. Furthermore, the predictors related to oral hygiene behavior were also determined. It was found that the recruits held fairly favorable attitudes toward oral hygiene behavior and generally felt in control of performing this behavior. The associations of these factors with intention and oral hygiene behavior varied, with multivariate analyses showing perceived behavior control to be strongly associated with intention to perform oral hygiene behavior and not with actual oral hygiene behavior. A regression analysis indicated that perceived behavior control was the best predictor of intention to perform oral hygiene behavior and explained, together with attitude, $37.2 \%$ of the variance in intention to perform oral hygiene behavior. In contrast, for actual oral hygiene behavior attitude was the significant predictor, and only $7.1 \%$ of the variance was explained. This research highlights the relative importance of the TPB constructs for behavioral intention and actual behavior, and these associations should be considered when designing practical recommendations for improving oral hygiene behavior. For instance, to increase recruits' motivation or intention to perform optimal oral hygiene behavior, perceived behavior control seems to be the most important factor to influence, followed by attitudes. Both intention and attitude had significant association with actual oral hygiene behavior, suggesting both a motivational and a structural educational approach.

This study has some limitations that need to be addressed in future studies. First, the large proportion of male participants may have biased the results. Because of the selective sample of mainly adolescents, medium/low educated, and unmarried man-of-the-land forces, these findings cannot be completely generalized to the population of army recruits as a whole. As known from past studies, there are apparent differences in oral health behaviors across demographic variables (e.g., gender, age, and lifestyle) and socioeconomic status. ${ }^{13}$ Although this does not imply that the relationships between the variables differ in different populations, it is recommended that these results be replicated in different military groups and in diverse contexts, such as during military training, which is a simulation of a real battlefield situation.

Nevertheless, one may make some practical recommendations for clinical practice, based on these findings, which suggest that interventions should target especially recruits' perceptions of behavioral control when seeking to increase intentions to perform optimal oral hygiene behavior and promote actual oral hygiene behavior. This study may assist military oral health professionals working with recruits and professional soldiers in what are referred to be "the most dignified 
tasks" of such professionals, i.e., educating recruits and professional soldiers in oral health and changing individuals' oral hygiene behavior. ${ }^{14}$

\section{CONCLUSION}

In The Netherlands Armed Forces oral health has to meet minimum standards according to the NATO agreement. This implies that the possibility of oral health problems has to be minimized during the period of the operational commitment, e.g., the deployment. Beside this mandatory oral health standard, which will be primarily monitored and executed by the military dentist, there is also an explicit place for preventive measures. To maximize the oral hygiene behavior, the military oral health professionals have to be easily accessible. Dental hygienists constitute a subgroup in the oral health work force that is more easily accessible than dentists. In addition, dentists have taken little interest in advocacy to promote good oral health, preferring to treat rather than prevent oral diseases, ${ }^{15}$ so dental hygienists can promote desired oral hygiene behavior by adequate professional communication with the recruits.

\section{ACKNOWLEDGMENTS}

The authors thank Lt-Col Ad M.J. Wagemakers for allowing this research to be conducted and for his invaluable support. Special thanks to the recruits of the Schoolbat North of the post "J. W. F. Kazerne" in Assen for taking part in this study.

\section{APPENDIX 1}

Index for oral hygiene behavior (OHB)

The following questions are about your oral hygiene selfcare practices.

(1) How often do you brush your teeth?

\begin{tabular}{|ll|}
\hline$\square$ & Not every day \\
$\square$ & Once a day \\
$\square$ & Twice a day \\
$\square$ & More than 2 times a day \\
\hline
\end{tabular}

(2) When do you brush your teeth?

\begin{tabular}{|lllll|}
\hline Morning before breakfast & $\square$ & Yes & $\square$ & No \\
Morning after breakfast & $\square$ & Yes & $\square$ & No \\
Noon & $\square$ & Yes & $\square$ & No \\
After dinner in the evening & $\square$ & Yes & $\square$ & No \\
Before going to sleep & $\square$ & Yes & $\square$ & No \\
\hline
\end{tabular}

(3) How do you brush your teeth?

I brush my teeth

\begin{tabular}{|lcccccccc|}
\hline Softly & 1 & 2 & 3 & 4 & 5 & 6 & 7 & Forcefully \\
& $\square$ & $\square$ & $\square$ & $\square$ & $\square$ & $\square$ & $\square$ & \\
\hline
\end{tabular}

(4) How much time do you spend on brushing your teeth? I brush my teeth

\begin{tabular}{|ll|}
\hline$\square$ & Shorter than 1 minute \\
$\square$ & 1 minute \\
$\square$ & 2 minutes \\
$\square$ & 3 minutes \\
$\square$ & Longer than 3 minutes \\
\hline
\end{tabular}

(5) I brush my teeth as follows:

\begin{tabular}{|c|c|}
\hline$\square$ & Back and forth movement ("horizontal" method) \\
\hline$\square$ & Up and down movement ("vertical" method) \\
\hline$\square$ & Circular movement ("circular" method) \\
\hline$\square$ & $\begin{array}{l}\text { Brushing softly with a massing movement near } \\
\text { the gum ("Bass" method) }\end{array}$ \\
\hline
\end{tabular}

(6) What do you use to clean your teeth?

Mostly I use:

\begin{tabular}{|ll|}
\hline$\square$ & Toothpaste with fluoride \\
$\square$ & Toothpaste without fluoride \\
$\square$ & I don't know \\
\hline
\end{tabular}

(7) Do you clean your tongue?

\begin{tabular}{|ll|}
\hline$\square$ & Never \\
$\square$ & Sometimes \\
$\square$ & Every day \\
\hline
\end{tabular}

(8) Which of the following interdental tools do you use?

\begin{tabular}{|c|c|c|c|c|}
\hline & Never & $\begin{array}{c}\text { Not } \\
\text { every day }\end{array}$ & $\begin{array}{c}\text { Once a } \\
\text { day }\end{array}$ & $\begin{array}{c}\text { Twice or more } \\
\text { times a day }\end{array}$ \\
\hline Floss & $\square$ & $\square$ & $\square$ & $\square$ \\
\hline Tooth sticks & $\square$ & $\square$ & $\square$ & $\square$ \\
\hline $\begin{array}{c}\text { Interdental } \\
\text { brushes }\end{array}$ & $\square$ & $\square$ & $\square$ & $\square$ \\
\hline
\end{tabular}

\section{REFERENCES}

1. Škec V, Špiček J, Sušac M, Jokić D, Brajdić D, Macan D: Influence of oral hygiene on oral health of recruits and professionals in the Croatian Army. Mil Med 2006; 171: 1006-9.

2. Van der Meer R, Schwamborn V: The Dutch Military Medical Services 'A bloody good bunch of professionals'. Medical Corps International Forum 2008; 3: 4-7.

3. Huis in 't Veld JHJ, van Palenstein Helderman WH, Backer Dirks O: Streptococcus mutans and dental caries in humans: a bacteriological and immunological study. Antonie van Leeuwenhoek 1979; 45: 25-33.

4. Ajzen I: Attitudes, personality, and behavior. Homewood, Il. US: Dorsey Press, 1988.

5. Ajzen I: The theory of planned behavior. Organ Behav Hum Decis Process 1991; 50: 179-211. 
6. Godin G, Kok G: The theory of planned behavior: a review of its application to health related behaviors. Am J Health Promot 1996; 11: 87-98.

7. Armitage CJ, Conner M: Efficacy of the theory of planned behavior: a meta-analytic review. Br J Soc Psychol 2001; 40: 471-99.

8. Smith JM: An evaluation of the applicability of the RosenstockHochbaum health behaviour model to the prevention of periodontal disease in English school girls. J Clin Periodontol 1974; 1: 222-31.

9. Stokes E, Ashcroft A, Platt MJ: Determining Liverpool adolescents' beliefs and attitudes in relation to oral health. Health Educ Res 2006; 21(2): 192-205.

10. Richard ND, Cohen LK (eds): Social sciences and dentistry. A critical bibliography. FDI, 1971.
11. Freeman R, Linden G: Health directed and health related dimensions of oral health behaviours of periodontal referrals. Community Dent Health 1995; 12: 48-51.

12. ADA: (2007). Cleaning your teeth and gums (oral hygiene). Available at http://www.ada.org/public/topics/cleaning_faq.asp\#4; accessed September 26, 2007.

13. Sakki TK, Knuuttila ML, Antilla SS: Lifestyle, gender and occupational status as determinants of dental health behavior. J Clin Periodontol 1998; 25: $566-70$.

14. Özcan M: Clinical Dental Biomaterials: Dentists Wealthy-Patients Healthy? Groningen, Facilitair Bedrijf, University of Groningen, 2008.

15. The Lancet Editorial: Oral health: prevention is key. Available at www. thelancet.com Vol 373 No 9657; accessed January 3, 2009. 